\title{
On the convexity of Relativistic Hydrodynamics
}

\author{
José María Ibáñez ${ }^{1}$, Isabel Cordero-Carrión ${ }^{2}$, José María \\ Martí ${ }^{1}$ and Juan Antonio Miralles ${ }^{3}$ \\ ${ }^{1}$ Department of Astronomy and Astrophysics, University of Valencia, E-46100, \\ Burjassot, Spain \\ 2 Max-Planck Institute for Astrophysics, Garching, Karl-Schwarzschild-Str. 1, \\ D-85741, Garching, Germany \\ ${ }^{3}$ Department of Applied Physics, University of Alicante, Campus de Sant Vicent del \\ Raspeig, E-03080, Alicante, Spain \\ E-mail: jose.m.ibanez@uv.es, chabela@mpa-garching.mpg.de, \\ Jose-Maria.Marti@uv.es, ja.miralles@ua.es
}

\begin{abstract}
The relativistic hydrodynamic system of equations for a perfect fluid obeying a causal equation of state is hyperbolic [1]. In this report, we derive the conditions for this system to be convex in terms of the fundamental derivative of the equation of state [2]. The classical limit is recovered.
\end{abstract}

PACS numbers: 04.25.D-, 47.11.-j, 47.75.+f, 95.30.Sf 
Recent applications in several fields of relativistic astrophysics demand the use of realistic equations of state (EOS) beyond the polytropic and ideal gas EOS. In this work, we report on the characterization of convexity of general relativistic hydrodynamics (GRHD) and, more specifically, on the conditions imposed by thermodynamics.

Let $\frac{\partial u}{\partial t}+\frac{\partial f(u)}{\partial x}=0$ be a scalar non-linear conservation law (in one spatial dimension, 1D) for the conserved variable $u: \mathbb{R} \times \mathbb{R} \longrightarrow \mathbb{R}, u=u(x, t), f: \mathbb{R} \longrightarrow$ $\mathbb{R}, f=f(u)$ being a non-linear function of $u(f l u x)$. The behaviour of $f(u)$ has important consequences on the behaviour of the solution $u(x, t)$ of the conservation law itself [3]. A crucial property is the monotonicity of the characteristic speed $\lambda(u)=$ $d f / d u=f^{\prime}(u)$. A convex (concave) flux satisfies $\lambda^{\prime}(u)=d \lambda / d u=f^{\prime \prime}(u)>0(<0)$, $\forall u$. If $\lambda(u)$ has extrema, i.e., $\exists u$ such that $\lambda^{\prime}(u)=d \lambda / d u=f^{\prime \prime}(u)=0$, then the conservation law describes a non-convex, non-concave flux.

Let us now consider a non-linear, hyperbolic system of conservation laws (HSCL) of $p$ equations in $1 \mathrm{D}$

$$
\frac{\partial \mathbf{u}}{\partial t}+\frac{\partial \mathbf{f}(\mathbf{u})}{\partial x}=0
$$

where $\mathbf{u}: \mathbb{R} \times \mathbb{R} \longrightarrow \mathbb{R}^{p}, \mathbf{u}=\mathbf{u}(x, t)$ is the vector of conserved variables, and $f: \mathbb{R}^{p} \longrightarrow \mathbb{R}^{p}, \mathbf{f}=\mathbf{f}(\mathbf{u})$, a non-linear function of $\mathbf{u}$, the vector of fluxes. Let $\lambda_{\alpha}(\mathbf{u})$ be the (real) eigenvalues and $\mathbf{r}_{\alpha}(\mathbf{u})(\alpha=1,2, \ldots, p)$ the corresponding right eigenvectors of the Jacobian matrix $\frac{\partial \mathbf{f}}{\partial \mathbf{u}}$.

A characteristic field $\mathcal{C}_{\alpha}$ of system (11) satisfying

$$
\mathcal{C}_{\alpha}: \frac{d x}{d t}=\lambda_{\alpha}
$$

is said to be genuinely nonlinear or linearly degenerate if, respectively,

$$
\begin{aligned}
& \mathcal{P}_{\alpha}:=\vec{\nabla}_{\mathbf{u}} \lambda_{\alpha} \cdot \mathbf{r}_{\alpha} \neq 0 \\
& \mathcal{P}_{\alpha}:=\vec{\nabla}_{\mathbf{u}} \lambda_{\alpha} \cdot \mathbf{r}_{\alpha}=0
\end{aligned}
$$

for all $\mathbf{u}$, where $\vec{\nabla}_{\mathbf{u}} \lambda_{\alpha}$ is the gradient of $\lambda_{\alpha}(\mathbf{u})$ in the space of conserved variables and the dot stands for the inner product in $\mathbb{R}^{p}$.

Conditions (3) and (4), introduced by Lax [4] are the extension to systems of the above property on monotonicity of the characteristic velocity described for the scalar case [3], and the HSCL is said to be convex (comprising both convex and concave fluxes in the scalar case) if all its characteristic fields are either genuinely non-linear or linearly degenerate. Additionally, in a non-convex system, non-convexity is associated with those states $\mathbf{u}$ for which one $\mathcal{P}_{\alpha}$ is zero and changes sign in a neighbourhood of $\mathbf{u}$.

In classical fluid dynamics, the convexity of the system is determined by the EOS [2, 3] and, more specifically, by the so-called fundamental derivative, $\mathcal{G}$ (see its definition 
and properties in, e.g., [2])

$$
\mathcal{G}:=-\frac{1}{2} V \frac{\left.\frac{\partial^{2} p}{\partial V^{2}}\right|_{s}}{\left.\frac{\partial p}{\partial V}\right|_{s}}
$$

$p$ being the pressure, $V:=1 / \rho$ the specific volume ( $\rho$ is the rest-mass density) and $s$ the specific entropy. An alternative expression for $\mathcal{G}$ is $[2]$

$$
\mathcal{G}=1+\left.\frac{\partial \ln c_{s, c l}}{\partial \ln \rho}\right|_{s},
$$

where $c_{s, c l}:=\sqrt{\partial p /\left.\partial \rho\right|_{s}}$ is the classical (i.e., non-relativistic) definition of the speed of sound.

It is important to note, however, that in fluid dynamics, one speaks of convexity when the thermodynamics forces rarefaction waves to be expansivet. In practice, this corresponds to impose the sign of $\mathcal{G}$ and, as we shall see below and in the Appendix, also the one of the $\mathcal{P}_{\alpha}$ associated to the genuinely nonlinear fields. The fundamental derivative measures the convexity of the isentropes in the $p-V$ plane and if $\mathcal{G}>0$ then the isentropes in the $p-V$ plane are convex and the rarefaction waves are expansive. Then, one speaks of convex EOS and the result is a convex system, with characteristic fields which are either genuinely nonlinear or linearly degenerate, and definite signs for the $\mathcal{P}_{\alpha}$ associated with the genuinely nonlinear fields. Conversely, a non-convex EOS (in which $\mathcal{G}<0$ or has not a definite sign) leads to a non-convex flow dynamicd\}.

The main goal of this report is to characterize the convexity of GRHD by examining the products $\mathcal{P}_{\alpha}$ defined in (3) and (4) (Lax's criterion). From this analysis, the convexity will be described in terms of a new condition on $\mathcal{G}$ that generalizes the classical Menikoff-Plohr's result [2]. The Appendix establishes the same condition on $\mathcal{G}$ but obtained following Menikoff-Plohr's approach applied to self-similar relativistic flows in $1 \mathrm{D}$, and discusses the connection of this result with the choice of the signs of $\mathcal{P}_{\alpha}$ associated with the genuinely nonlinear fields. Our results generalize those outlined in [6].

The evolution of a relativistic fluid is governed by a set of conservation laws, namely the conservation of rest mass, $\nabla \cdot \mathbf{J}=0$, and the conservation of energy-momentum, $\nabla \cdot \mathbf{T}=0(\nabla \cdot$ stands for the four-divergence). For a perfect fluid, the components of the rest-mass current, $\mathbf{J}$, and the energy-momentum tensor, $\mathbf{T}$, are $J^{\mu}=\rho u^{\mu}$, and $T^{\mu \nu}=\rho h u^{\mu} u^{\nu}+p g^{\mu \nu}$, respectively, $h$ being the specific enthalpy, defined by $h=1+\varepsilon+p / \rho$, where $\varepsilon$ is the specific internal energy. $u^{\mu}$ is the four-velocity of the fluid and $g_{\mu \nu}$ defines the metric of the spacetime $\mathcal{M}$ where the fluid evolves. Greek (Latin)

$\ddagger$ A rarefaction wave is said to be expansive when fluid elements decrease their pressure and density as they go through it.

$\S$ However, note that for more general HSCLs, there can be non-convex states even for a convex EOS, as in the case of classical ideal magnetohydrodynamics [5]. 
indices run from 0 to 3 (1 to 3), or, alternatively, they stand for general coordinates $\{t, x, y, z\}(\{x, y, z\})$. The geometrized system of units $(c=G=1)$ and the summation convention over repeated indices are used.

An $\operatorname{EOS~} p=p(\rho, \varepsilon)$ closes the system. Accordingly, the (relativistic) sound speed $c_{s}:=\sqrt{\partial p /\left.\partial e\right|_{s}}, e:=\rho(1+\varepsilon)$, satisfies $h c_{s}^{2}=\chi+\frac{p}{\rho^{2}} \kappa$, with $\chi:=\partial p /\left.\partial \rho\right|_{\varepsilon}$ and $\kappa:=\partial p /\left.\partial \varepsilon\right|_{\rho}$.

In [7], the equations of GRHD were written as a hyperbolic system of conservation laws within the framework of the $\{3+1\}$ formalism (see, e.g., [8]). According to this formalism, the metric is split into the objects $\alpha$ (lapse), $\beta^{i}$ (shift) and $\gamma_{i j}$, keeping the line element in the form:

$$
d s^{2}=-\left(\alpha^{2}-\beta_{i} \beta^{i}\right) d t^{2}+2 \beta_{i} d x^{i} d t+\gamma_{i j} d x^{i} d x^{j}
$$

If $\mathbf{n}$ is a unit timelike vector field normal to the spacelike hypersurfaces $\Sigma_{t}(\mathrm{t}=$ const.), then, by definition of $\alpha$ and $\beta^{i}, \partial_{t}=\alpha \mathbf{n}+\beta^{i} \partial_{i}$, with $\mathbf{n} \cdot \partial_{i}=0, \forall i$. Observers, $\mathcal{O}_{E}$, at rest in the slice $\Sigma_{t}$, i.e., those having $\mathbf{n}$ as four-velocity (Eulerian observers), measure the following velocity of the fluid:

$$
v^{i}=\frac{u^{i}}{\alpha u^{t}}+\frac{\beta^{i}}{\alpha}
$$

where $W:=-\left(u^{\mu} n_{\mu}\right)=\alpha u^{t}$, the Lorentz factor, satisfies $W=\left(1-\mathrm{v}^{2}\right)^{-1 / 2}$, with $\mathrm{v}^{2}=v_{i} v^{i}\left(v_{i}=\gamma_{i j} v^{j}\right)$.

The set of conserved variables gathers those quantities which are directly measured by $\mathcal{O}_{E}$, i.e., the rest-mass density, $D$, the momentum density in the $j$-direction, $S_{j}$, and the total energy density, $E$. In terms of the primitive variables $\mathbf{w}=\left(\rho, v_{i}, \varepsilon\right)$, they are

$$
D=\rho W, \quad S_{j}=\rho h W^{2} v_{j}, \quad E=\rho h W^{2}-p .
$$

With the above definitions, the fundamental first-order, flux-conservative system ruling the evolution of flows in a given spacetime (GRHD equations) reads

$$
\frac{1}{\sqrt{-g}}\left(\frac{\partial \sqrt{\gamma} \mathbf{F}^{0}(\mathbf{w})}{\partial x^{0}}+\frac{\partial \sqrt{-g} \mathbf{F}^{i}(\mathbf{w})}{\partial x^{i}}\right)=\mathbf{s}(\mathbf{w}),
$$

where

$$
\mathbf{u}:=\mathbf{F}^{0}(\mathbf{w})=\left(D, S_{j}, \tau\right)
$$

is the vector of conserved variables, where $\tau:=E-D$ (total energy density excluding the rest-mass one),

$$
\mathbf{F}^{i}(\mathbf{w})=\left(D\left(v^{i}-\frac{\beta^{i}}{\alpha}\right), S_{j}\left(v^{i}-\frac{\beta^{i}}{\alpha}\right)+p \delta_{j}^{i}, \tau\left(v^{i}-\frac{\beta^{i}}{\alpha}\right)+p v^{i}\right)
$$

are the fluxes in each spatial direction, and the corresponding sources $\mathbf{s}(\mathbf{w})$ are 


$$
\mathbf{s}(\mathbf{w})=\left(0, T^{\mu \nu}\left(\frac{\partial g_{\nu j}}{\partial x^{\mu}}-\Gamma_{\nu \mu}^{\delta} g_{\delta j}\right), \alpha\left(T^{\mu 0} \frac{\partial \ln \alpha}{\partial x^{\mu}}-T^{\mu \nu} \Gamma_{\nu \mu}^{0}\right)\right)
$$

In the previous expressions, $g:=\operatorname{det}\left(g_{\mu \nu}\right)$ and $\gamma:=\operatorname{det}\left(\gamma_{i j}\right)$, and are such that $\sqrt{-g}=\alpha \sqrt{\gamma}$

The three $5 \times 5$ - Jacobian matrices $\mathcal{B}^{i}$ associated with system (10) are

$$
\mathcal{B}^{i}=\alpha \frac{\partial \mathbf{F}^{i}}{\partial \mathbf{F}^{0}}
$$

The full spectral decomposition $\left\{\lambda_{n}, \mathbf{r}_{n}\right\}^{(i)}(n=1, \ldots, 5)$ of the above matrices $\mathcal{B}^{i}$ can be found in [9] ․ In terms of the primitive variables $\mathbf{w}$, we can define the following matrices

$$
\mathcal{A}^{i}=\alpha \frac{\partial \mathbf{F}^{i}(\mathbf{w})}{\partial \mathbf{w}}, \quad \mathcal{A}^{0}=\frac{\partial \mathbf{F}^{0}(\mathbf{w})}{\partial \mathbf{w}} .
$$

It has been shown in [10] that:

a) The matrices $\mathcal{A}^{i}$ and $\mathcal{B}^{i}$ satisfy $\mathcal{B}^{i}=\mathcal{A}^{i}\left(\mathcal{A}^{0}\right)^{-1}$

b) If $\left\{\lambda_{n}^{*}, \mathbf{r}_{n}^{*}\right\}^{(i)}(n=1, \ldots, 5)$ are, respectively, the eigenvalues and eigenvectors of the characteristic eigenvalue problem corresponding to the system written in quasilinear form, $\left(\mathcal{A}^{i}-\lambda^{*} \mathcal{A}^{0}\right) \mathbf{r}^{*}=0$, then the following relations are satisfied

$$
\left\{\lambda_{n}, \mathbf{r}_{n}\right\}^{(i)}=\left\{\lambda_{n}^{*}, \mathcal{A}^{0} \mathbf{r}_{n}^{*}\right\}^{(i)}(n=1, \ldots, 5)
$$

On the other hand,

Proposition 1. Given an arbitrary 3-vector $\zeta_{i}$, the general eigenvalue problem $\left(\zeta_{j} \mathcal{A}^{j}-\lambda \mathcal{A}^{0}\right) \mathbf{r}^{*}=0$ has the following eigenvalues and right eigenvectors:

- $\lambda_{0}=\alpha \zeta_{j}\left(v^{j}-\frac{\beta^{j}}{\alpha}\right)$, degenerate (triple) eigenvalue associated with the material waves;

- $\lambda_{ \pm}=-\zeta_{j} \beta^{j}+\frac{\alpha}{1-\mathrm{v}^{2} c_{s}^{2}}\left[\left(\zeta_{j} v^{j}\right)\left(1-c_{s}^{2}\right) \pm c_{s} W^{-1} \sqrt{\left(1-\mathrm{v}^{2} c_{s}^{2}\right)\left(\zeta_{j} \zeta^{j}\right)-\left(1-c_{s}^{2}\right)\left(\zeta_{j} v^{j}\right)^{2}}\right]$ associated with the acoustic waves;

- $\mathbf{r}_{0, m}^{*}=\left(-\kappa, \zeta_{m, i}^{\perp}, \chi\right)$, where $m(=1,2,3)$ identifies the three independent right eigenvectors associated to $\lambda_{0}$, and $\zeta_{m, i}^{\perp}(m=1,2,3)$ stands for the zero vector and the two unitary 3-vectors forming an orthonormal basis with $\zeta_{i} / \sqrt{\zeta_{j} \zeta^{j}}$;

- $\mathbf{r}_{ \pm}^{*}=\left(\rho \kappa \Delta_{ \pm}, \kappa\left(\zeta_{j} v^{j}-\Lambda_{ \pm}\right)\left(\zeta_{i}-\Lambda_{ \pm} v_{i}\right),-\rho \chi \Delta_{ \pm}-\rho h W^{2}\left(\zeta_{j} v^{j}-\Lambda_{ \pm}\right)^{2}\right)$, with $\Delta_{ \pm}=-W^{2}\left(\Lambda_{ \pm}-\zeta_{j} v^{j}\right)^{2}-\left(\zeta_{j} \zeta^{j}\right)+\Lambda_{ \pm}^{2}$, and $\Lambda_{ \pm}=\left(\lambda_{ \pm}+\zeta_{j} \beta^{j}\right) / \alpha$.

|| The right-eigenvectors published in [7] correspond to those spacetimes having a diagonal metric. 
The spectral decomposition in the $j$ spatial direction is recovered by taking $\zeta_{i}=\delta_{i}^{j}$ and, therefore, $\zeta^{i}=\gamma^{i k} \delta_{k}^{j}=\gamma^{i j}$. The special relativistic limit (Minkowski spacetime) of the above expressions is recovered (in Cartesian coordinates) just doing $\alpha=1, \beta^{i}=0, \gamma_{i j}=\delta_{i j}$.

Finally, taking into account that

$$
\vec{\nabla}_{\mathbf{u}} \lambda_{\alpha}(\mathbf{u}) \cdot \mathbf{r}_{\alpha}(\mathbf{u}) \neq 0 \Longleftrightarrow \vec{\nabla}_{\mathbf{w}} \lambda_{\alpha}(\mathbf{w}) \cdot \mathbf{r}^{*}{ }_{\alpha}(\mathbf{w}) \neq 0
$$

we can formulate the following proposition:

Proposition 2. The quantity $\mathcal{P}_{ \pm}$, in GRHD, can be given in terms of three factors, one coming from the particular renormalization procedure followed to get the eigenvectors, $\mathcal{R}_{ \pm}$, a kinematical one, $\mathcal{K}_{ \pm}$, and a purely thermodynamical one, $\mathcal{T}:$

$$
\mathcal{P}_{ \pm}=\mathcal{R}_{ \pm} \mathcal{K}_{ \pm} \mathcal{T}
$$

where $\mathcal{R}_{ \pm}=\alpha \rho \kappa \Delta_{ \pm}$. The kinematical term is:

$$
\mathcal{K}_{ \pm}\left(\zeta_{j}, \gamma_{i j}, v_{j}\right)= \pm \frac{W^{2}}{\delta}\left(\frac{\left(\zeta_{j} \zeta^{j}\right)-\left(\zeta_{j} v^{j}\right)^{2}}{c_{s}\left(\zeta_{j} v^{j}\right) \pm \delta}\right)^{2}
$$

with

$$
\delta=W\left[\left(1-\mathrm{v}^{2} c_{s}^{2}\right)\left(\zeta_{j} \zeta^{j}\right)-\left(1-c_{s}^{2}\right)\left(\zeta_{j} v^{j}\right)^{2}\right]^{1 / 2}
$$

The thermodynamical term is:

$$
\mathcal{T}(\rho, \varepsilon)=\frac{\partial c_{s}}{\partial \rho}+\frac{p}{\rho^{2}} \frac{\partial c_{s}}{\partial \varepsilon}+\frac{c_{s}}{\rho}\left(1-c_{s}^{2}\right) .
$$

The term $\mathcal{R}_{ \pm} \mathcal{K}_{ \pm}$has the same sign for all the states $(\mathbf{u})$ of the system. It only tends to zero when $\alpha \rightarrow 0, W \rightarrow \infty$ (ultrarelativistic flow speeds), or $c_{s} \rightarrow 1$ (ultrarelativistic thermodynamics).

The thermodynamical term in $\mathcal{P}_{ \pm}, \mathcal{T}$, can be written, in a more compact way, as a function of the fundamental derivative, $\mathcal{G}$ (see Eq. (5) )

$$
\mathcal{T}=\frac{c_{s}}{\rho}\left(\mathcal{G}-\frac{3}{2} c_{s}^{2}\right)
$$

This formula has been derived considering: i) the definition of $\mathcal{G}$ given in terms of the classical speed of sound (see Eq. (6 6 ) ), ii) the relationship between both definitions of the local speed of sound, $c_{s, c l}^{2}=h c_{s}^{2}$, from which it is easy to show

$$
\left.\frac{\partial \ln c_{s, c l}}{\partial \ln \rho}\right|_{s}=\left.\frac{\partial \ln c_{s}}{\partial \ln \rho}\right|_{s}+\frac{1}{2} c_{s}^{2}
$$

and iii) the thermodynamical partial derivative:

$$
\left.\frac{\partial \mathcal{Q}}{\partial \rho}\right|_{s}=\left.\frac{\partial \mathcal{Q}}{\partial \rho}\right|_{\varepsilon}+\left.\frac{p}{\rho^{2}} \frac{\partial \mathcal{Q}}{\partial \varepsilon}\right|_{\rho}
$$


where $\mathcal{Q}$ stands for any thermodynamical function of $\rho, \varepsilon$. Hence, as far as the thermodynamical term is concerned, Eq. (21) on one hand, and Eqs. (33), (34) of the Appendix on the other, allows us to establish the following corollary:

Corollary 1. Unlike classical fluid dynamics, which is convex for $\mathcal{G}>0$, GRHD is convex when the inequality $\mathcal{G}>\frac{3}{2} c_{s}^{2}$ is satisfied.

Alternatively, in terms of the (relativistic) fundamental derivative $\tilde{\mathcal{G}}$, GRHD is convex when

$$
\tilde{\mathcal{G}}:=-\frac{1}{2} \frac{h}{\rho}\left(1-c_{s}^{2}\right)^{2} \frac{\left.\frac{\partial^{2} p}{\partial(h / \rho)^{2}}\right|_{s}}{\left.\frac{\partial p}{\partial(h / \rho)}\right|_{s}}>0 .
$$

It can be easily seen that for causal (i.e., $\left.c_{s}^{2}<1\right)$ EOS, the derivative $\partial p /\left.\partial(h / \rho)\right|_{s}$, appearing in the denominator of the definition of $\tilde{\mathcal{G}}$, must be negative. Hence, for causal EOS, the convexity condition expressed in (23) translates to $\partial^{2} p /\left.\partial(h / \rho)^{2}\right|_{s}>0$. Israel [1] proved that $\partial^{2}(h / \rho) /\left.\partial p^{2}\right|_{s}>0$ is a necessary condition for the stability of compressive shocks and the non-occurrence of rarefactive shocks. In the same context, Lichnerowicz [12] showed that the compressibility conditions $\partial(h / \rho) /\left.\partial p\right|_{s}<0$, $\partial(h / \rho) /\left.\partial s\right|_{p}>0$ and $\partial^{2}(h / \rho) /\left.\partial p^{2}\right|_{s}>0$ are sufficient conditions for a shock to be compressive. Lately, Thorne [13] (see also [11]) proved the same result as Lichnerowicz eliminating the second condition and only for weak shocks. Taking into account the nature of shocks as limiting simple waves we can conclude that our result, expressed in Corollary 1, comprises these early results on the characterization of compressive shocks.

It would be interesting to probe the present result in numerical experiments and astrophysical applications using complex EOS.

\section{Acknowledgments}

Work partially supported by the Spanish Ministry of Science (grants: AYA201021097-C03-01 and AYA2010-21097-C03-2), and a PROMETEO-2009-103 grant from the Local Government of the Valencian Community. I. C.-C. acknowledges support from Alexander von Humboldt Foundation.

\section{Appendix. Characterizing the RHD convexity through Menikoff-Plohr's approach and the connection with Lax's criterion}

The SRHD (special relativistic hydrodynamics) equations for mass and momentum conservation in $1 \mathrm{D}$ are respectively

$$
\frac{\partial(\rho W)}{\partial t}+\frac{\partial(\rho W v)}{\partial x}=0,
$$


On the convexity of Relativistic Hydrodynamics

$$
\frac{\partial\left(\rho h W^{2} v\right)}{\partial t}+\frac{\partial\left(\rho h W^{2} v^{2}+p\right)}{\partial x}=0
$$

(where $v$ stands for the flow velocity along the $x$ direction, and the rest of quantities were already defined).

Self-similar solutions of the above equations for isentropic flow $\left(d p=h c_{s}^{2} d \rho\right)$ are obtained by imposing that all variables are functions of $\xi=x / t$. The following relations between the differentials are easily obtained:

$$
\begin{aligned}
& (v-\xi) d p+\rho h c_{s}^{2}\left[1+v W^{2}(v-\xi)\right] d v=0, \\
& {\left[1+v W^{2}(v-\xi)\right] d p+\rho h W^{2}(v-\xi) d v=0 .}
\end{aligned}
$$

Eliminating the differentials, the following condition has to be fulfilled

$$
(v-\xi) W^{2}=\mp c_{s}\left[1+v W^{2}(v-\xi)\right],
$$

which can be used to obtain the relation between the differentials $d p$ and $d v$,

$$
d p= \pm \rho h W^{2} c_{s} d v .
$$

Equation (28) allows us to write $\xi$ as

$$
\xi_{ \pm}=\frac{v \pm c_{s}}{1 \pm v c_{s}}
$$

Now, differentiating the previous expression and using the thermodynamic relation $d h=d p / \rho$, valid for isentropic flow, it is straightforward to show that

$$
d \xi_{ \pm}= \pm \frac{1-v^{2}}{\left(1 \pm v c_{s}\right)^{2}} \tilde{\mathcal{G}} \frac{d p}{\rho h c_{s}}
$$

where

$$
\tilde{\mathcal{G}}=1+\left.\frac{\partial \ln c_{s}}{\partial \ln \rho}\right|_{s}-c_{s}^{2},
$$

which can be easily written in terms of the fundamental derivative, $\mathcal{G}$, using Eqs. (6) and (22)

$$
\tilde{\mathcal{G}}=\mathcal{G}-\frac{3}{2} c_{s}^{2}
$$

At this point, it is important to note that $\tilde{\mathcal{G}}$ can be written as

$$
\tilde{\mathcal{G}}=-\frac{1}{2} \frac{h}{\rho}\left(1-c_{s}^{2}\right)^{2} \frac{\left.\frac{\partial^{2} p}{\partial(h / \rho)^{2}}\right|_{s}}{\left.\frac{\partial p}{\partial(h / \rho)}\right|_{s}}
$$

in complete correspondence with the definition of $\mathcal{G}$, (51).

Convexity (i.e., the fact that rarefaction fans are expansive) implies that the derivative $d \xi_{+} / d p$ must be positive, and the derivative $d \xi_{-} / d p$ must be negative [2] forcing $\tilde{\mathcal{G}}$ to be positive (or $\mathcal{G}>\frac{3}{2} c_{s}^{2}$ ). In the opposite case, i.e., $d \xi_{+} / d p<0$ and 
$d \xi_{-} / d p>0$, rarefaction waves are compressive. If the sign of these derivatives is not defined and $d \xi_{ \pm} / d p$ is positive for some states and negative for others the acoustic waves lose the genuinely non-linear character.

All the above analysis can be done in a compact way by using the spectral decomposition of the 1D SRHD system. Particularizing the spectral decomposition presented in Proposition 1 to this case, the corresponding eigenvalue problem in terms of the primitive variables $\mathbf{w}=(\rho, v, \varepsilon)$ leads to the following eigenvalues

$$
\lambda_{0}=v, \quad \lambda_{ \pm}=\frac{v \pm c_{s}}{1 \pm v c_{s}}
$$

and eigenvectors

$$
\begin{aligned}
& \mathbf{r}_{0}^{*}=(-\kappa, 0, \chi), \\
& \mathbf{r}_{ \pm}^{*}=\left(\frac{1}{h c_{s}^{2}}, \pm \frac{1}{\rho h c_{s} W^{2}}, \frac{p}{\rho^{2} h c_{s}^{2}}\right)
\end{aligned}
$$

where the normalization of the eigenvectors $\mathbf{r}_{ \pm}^{*}$ has been chosen so that its first component, $r_{ \pm, 1}^{*}$, fulfills the equation

$$
\frac{d \rho}{r_{ \pm, 1}^{*}}=d p
$$

for isentropic flows.

Now, taking into account that, in a self-similar flow, the self-similar variable is $\xi_{ \pm}=\lambda_{ \pm}$and the following relation between the variation of the variables across the flow and the components of the right eigenvectors holds,

$$
\frac{d \rho}{r_{ \pm, 1}^{*}}=\frac{d v}{r_{ \pm, 2}^{*}}=\frac{d \varepsilon}{r_{ \pm, 3}^{*}}
$$

the previous election allows us to write the differential of the eigenvalues $\lambda_{ \pm}$as

$$
d \lambda_{ \pm}=\vec{\nabla}_{\mathbf{w}} \lambda_{ \pm}(\mathbf{w}) \cdot d \mathbf{w}=\vec{\nabla}_{\mathbf{w}} \lambda_{ \pm} \cdot \mathbf{r}_{ \pm}^{*} d p
$$

Therefore, the sign of $\vec{\nabla}_{\mathbf{w}} \lambda_{ \pm} \cdot \mathbf{r}_{ \pm}^{*}$ determines the character of the flow. Convexity (expansive rarefaction fans) is obtained for a positive value of $\vec{\nabla}_{\mathbf{w}} \lambda_{+} \cdot \mathbf{r}_{+}^{*}$ and a negative value of $\vec{\nabla}_{\mathrm{w}} \lambda_{-} \cdot \mathbf{r}_{-}^{*}$.

\section{References}

[1] Anile M A 1989 Relativistic fluids and magneto-fluids (Cambridge, England: Cambridge University Press)

[2] Menikoff R and Plohr B J 1989 Reviews of Modern Physics 6175

[3] Toro E F 2009 Riemann solvers and numerical methods for fluid dynamics: a practical introduction (Berlin, Germany: Springer, 3rd. edition) 
[4] Lax P D 1957 Comm. Pure Appl. Math. 10537

[5] Brio M and Wu C C 1988 J. Comput. Phys. 75400

[6] Ibáñez J Mㅁa, Aloy M A, Mimica P, Antón L, Miralles J A and Martí J Maa 2011 5th international conference of numerical modeling of space plasma flows (astronum 2010). Proceedings of a 5th international conference held at San Diego, California, USA 13-18 June 2010. San Francisco: Publications of the Astronomical Society of the Pacific, 2011, p.217

[7] Banyuls F, Font J A, Ibáñez J M므, Martí J Maa and Miralles J A 1997 Astrophys. J. 476221

[8] Gourgoulhon E $20123+1$ Formalism in General Relativity: Bases of Numerical Relativity, Lecture Notes in Physics 846 (Berlin, Germany: Springer)

[9] Ibáñez J M므, Aloy M A, Font J A, Martí J M므, Miralles J A and Pons J A 2001 Proc. from the Int. Conf. Godunov Methods: Theory and Applications (Oxford, United Kingdom) (New York: Kluwer Academic/Plenum Publishers, Ed. E. F. Toro)

[10] Font J A, Ibáñez J Mํㅡ, Marquina A and Martí J Mㅡㅡ 1994 Astron. Astrophys. 282304

[11] Israel W 1960 Proc. R. Soc. Lond. A 259129

[12] Lichnerowicz A 1967 Relativistic Hydrodynamics and Magnetohydrodynamics (New York, New York: Benjamin)

[13] Thorne K S 1973 Astrophys. J. 179897 